\title{
Construcción de una Escala de Habilidades Protomentalistas para infantes
}

\section{Building a Protomentalist Skills Scale for Infants}

\author{
Carmen Magali Melendez Jara \\ Universidad Nacional Mayor de San Marcos, Lima, Perú \\ ORCID: http://orcid.org/0000-0003-1446-4837
}

Recibido 28-05-19 Revisado 04-10-19 Aprobado 11-12-19 En línea 12-12-19

*Correspondencia

Email: carmen.melendez@unmsm.edu.pe
Citar como:

Melendez, C. (2019). Construcción de una Escala de Habilidades Protomentalistas para infantes. Propósitos y

Representaciones, $\quad 7(\mathrm{SPE}), \quad$ e337. doi: 


\section{Resumen}

Las habilidades protomentalistas son la base para desarrollar la teoría de la mente que es el componente cognitivo de la empatía, tan importante para el vínculo social efectivo de los seres humanos (Riviére, 2000; Sáiz, Carbonero \& Román, 2012; Westby \& Robinson, 2014). El objetivo del presente estudio fue construir una escala para la evaluación del desarrollo de Habilidades Protomentalistas (PROTO - HM) dirigido a infantes menores de 3 años y estimar su validez de contenido. La escala fue evaluada por 12 jueces expertos en desarrollo infantil de Estados Unidos, Chile y Perú, quienes observaron la claridad de los enunciados, así como la relevancia y la representatividad de los ítems según el modelo teórico propuesto. Para obtener la validez de contenido se utilizó la $\mathrm{V}$ de Aiken con intervalos de confianza al 95\%. Los resultados demuestran que todos los ítems, dimensiones y subdimensiones de las habilidades protomentalistas de interacción con las personas e interacción con los objetos presentan valores superiores a .80, siendo estos estadísticamente significativos respecto al criterio mínimo de Aiken (.60). A su vez evidencia un buen funcionamiento de ítems debido a que los límites inferiores del intervalo de confianza al 95\%, fueron mayores a .50 en todos los casos.

Palabras clave: Criterio de jueces; Habilidades protomentalistas; Interacción con personas; Interacción con objetos; Validez de contenido.

\section{Summary}

Protomentalist skills are the basis for developing the theory of mind, that is the cognitive component of empathy so important for the effective social bond of human beings (Riviére, 2000; Sáiz, Carbonero and Román, 2012; Westby and Robinson, 2014). The objectives of the present study were to build a scale for the evaluation of the development of Protomentalist skills (PROTO - HM) aimed at infants under the age of 3 , and to estimate its content validity. The scale was evaluated by 12 expert judges in child development in the United States of America, Chile and Peru. They observed the clarity of the statements, as well as the relevance and representativeness of the items according to the proposed theoretical model. In order to obtain the validity of content, Aiken $\mathrm{V}$ was used with 95\% confidence intervals. The results show that all the items, dimensions and sub-dimensions of the protomentalist skills of interaction with people and interaction with objects show values higher than .80 , these being statistically significant with respect to the minimum criteria of Aiken (.60). At the same time, it evidences a good item performance because the lower limits of the $95 \%$ confidence interval were greater than .50 in all cases.

Keywords: Judge Criteria; Protomentalist Skills; Interaction with People; Interaction with Objects; Content Validity.

\section{Introducción}

Las habilidades mentalistas se desarrollan durante la infancia y son conocidas también como teoría de la mente. Estas habilidades permiten la comprensión de que las personas no comparten los mismos pensamientos y sentimientos, que cada uno tiene su forma de pensar. Para Peterson, Wellman y Slaughter (2012) la teoría de la mente o habilidades mentalistas implican la "comprensión explícita de cómo el comportamiento humano se rige por los estados mentales de creencia, intención, memoria y deseo" (p. 469). Estas capacidades nos permiten "sintonizar" con las perspectivas de otras personas (Sussman, 2006) desarrollando de esta manera la empatía.

La capacidad empática, es la tendencia a estar psicológicamente en sintonía con los sentimientos y perspectivas de los demás y que nos permite vincularnos de forma eficiente (Decety \& Lamm, 2006). Davis (1994) reconoce que las sensibilidades empáticas son de naturaleza multidimensional y están conformadas por diversas dimensiones emocionales, tales como sentimientos de preocupación y compasión por los demás, y por dimensiones cognitivas o 
habilidades mentalistas como la capacidad para imaginar diferentes puntos de vista más allá de los propios.

En un estudio de Chopik, O’Brien y Konrath (2017) se exploró la variación cultural en la empatía y cómo esta variación estaba relacionada con las características psicológicas y el comportamiento prosocial en todas las culturas. Según los datos analizados de 104,365 adultos en 63 países diferentes, Ecuador fue el país con personas con mayor empatía en el mundo, seguido por Arabia Saudí y Perú. Pero, paradójicamente, según datos del Instituto Nacional de Estadística e Informática (INEI) del 2018, el Perú tiene uno de los índices más altos de violencia, y el 85.3 $\%$ de los peruanos mayores de 15 años se siente inseguro y el $22.2 \%$ ha sufrido conductas de amenaza e intimidación. Encontrándose a su vez, altos índices de incidencia de discriminación étnica y violencia de género. Estos comportamientos, contradicen los resultados debido a que no indican que seamos uno de los países más empáticos del planeta.

La empatía se aprende desde los primeros años de vida, para ello es importante estimularla desde edades tempranas. Estas habilidades no emergen de la noche a la mañana, sino que se desarrollan dentro de un patrón predecible y secuencial en el desarrollo neurotípico durante la infancia. Berk (2004) plantea hitos del desarrollo emocional en el niño, lo cual nos permite entender que los dos primeros años están llenos de interacciones psicosociales, todas resultantes de los genes, la maduración, la cultura y los cuidadores, que proporcionan un amplio abanico de emociones.

Las investigaciones apuntan que el desarrollo de las habilidades mentalistas se adquiere entre los cuatro y los cinco años (Berk, 2004). Sin embargo, existen habilidades mentalistas precursoras o habilidades protomentalistas que son necesarias para que se desarrolle la teoría de la mente o las habilidades mentalistas y estas surgen en la infancia temprana. Los bebés desde muy temprano en la vida encuentran a otras personas fascinantes e incluso antes de que puedan hablar, pueden interactuar y comunicarse con ellos. Tal interés e interacción dependen de formas tempranas de cognición social (Moore, 2010). Sin embargo, Astington y Edward (2010) advierten que existen problemas en la interpretación de cómo y cuándo los infantes desarrollan la cognición social. Algunos investigadores afirman que incluso los bebés son conscientes de los deseos y pensamientos de otras personas, mientras que otros piensan que esta comprensión no se desarrolla hasta los años preescolares. Para Astington y Edward (2010), esta contradicción se puede resolver tomando una visión evolutiva de la teoría de la mente, es decir, la conciencia intuitiva se vuelve más reflexiva y explícita, durante el desarrollo temprano y, además, el desarrollo de las habilidades lingüísticas de los niños juega un papel importante en esta transición.

\section{Habilidades protomentalistas}

Para Sáiz, Carbonero y Román (2012) las habilidades protomentalistas son aquellas precursoras a las actividades mentalistas o teoría de la mente, aspecto esencial para el desarrollo de la empatía, y que se activan antes de los tres años. Durante la primera infancia e infancia, los niños aprenden habilidades protomentalistas para la interacción con personas a través de la mediación social y para la interacción con objetos a través de la mediación instrumental, este último tipo de interacción marca el desarrollo de la representación mental.

Para Riviére (2000) durante el proceso de interacción social, los bebés aprenden a diferenciar entre los objetos y las personas. Descubren de manera progresiva que las personas hablan, se mueven, poseen un rostro e interaccionan y, sobre todo, que tienen mente y capacidad para relacionarse con otras mentes. Este conjunto de características, diferencian a las personas de los objetos. Por ello, la capacidad mental humana podría ser definida como la competencia de atribuir mente a otros, y que las creencias y los deseos, como entidades mentales, permiten predecir y comprender su conducta (Moore, 2010). 


\section{Dimensiones de las habilidades protomentalistas}

de Villiers y de Villiers y Westby y Robinson (2014), refieren que existen habilidades precursoras o habilidades protomentalistas que son necesarias que se desarrollen para que existan las habilidades mentalistas o la teoría de la mente y estas surgen en la infancia, antes de los 4 años. Las dimensiones protomentalistas se dividen en habilidades de interacción con las personas y habilidades de interacción con los objetos. Sáiz et al., (2012) realizan una propuesta:

Habilidades de interacción con las personas. Son capacidades que se producen por mediación social y que están conformadas por las relaciones triangulares, la intersubjetividad, el simbolismo y la referencia social.

- Relaciones triangulares: Evalúa la capacidad del niño para tener relaciones con los otros acerca de las cosas. Las relaciones comunicativas son, triangulares en el sentido de que implica tres componentes, "yo", "tú" y "el objeto" acerca del que versan. Las relaciones triangulares comprenden las siguientes habilidades: contacto ocular, atención, pautas de atención conjunta, uso del protoimperativo y del protodeclarativo.

- La intersubjetividad: Evalúa diversos modos en los cuales establecemos contacto con la subjetividad del otro, tiene que ver con tres aspectos: (a) primero, con el sentido de comunión interpersonal entre sujetos que ajustan tanto sus estados emocionales como sus expresiones respectivas uno a otro, (b) segundo con aquello que define la atención conjunta a objetos de referencia en un domino compartido de conversación lingüística o extra lingüística y (c) finalmente con la capacidad de inferencia acerca de las intenciones, creencias y sentimientos de otros, y que abarca la simulación o la capacidad para "leer" los estados mentales y procesos de los otros (remitiendo al concepto de empatía).

- El simbolismo: La función simbólica consiste, en "poder representar algo (un "significado" cualquiera: objeto, acontecimiento, esquema conceptual, etc.) por medio de un "significante" diferenciado y que sólo sirve para esa representación". El simbolismo comprende el juego simbólico y la imitación.

- La referencia social: Son formas de comunicación no verbal que implica buscar estímulos discriminativos provistos por otros sobre contingencias en un contexto ambiguo para responder de una manera que produzca refuerzo. Estas habilidades son: Manifestación, identificación y reconocimiento de emociones. Este tipo de aprendizajes se manifiestan en una clase de intercambios tríadicos denominados actos de referencia social.

Habilidades de interacción con los objetos. Evalúa la capacidad que surge gracias a la mediación instrumental y que posibilitan el desarrollo de la representación mental y están conformadas por la permanencia del objeto, la inteligencia práctica y la resolución de problemas con objetos y la relación entre los objetos (causalidad y lógica).

- Permanencia del objeto: Implica la existencia cognitiva del objeto en la mente del sujeto con independencia de su presencia real y de las acciones que sobre él se efectúen en el "aquí y en el ahora". Es decir, el sujeto, entiende que el objeto existe independientemente de su presencia física y de las acciones que sobre él se pueden efectuar.

- Inteligencia practica y resolución de problemas con objetos: El desarrollo de la inteligencia practica implica, además de la adquisición de la noción del objeto, la capacidad del sujeto de resolver problemas sin la utilización continua de las estrategias de ensayo - error y desde el inicio del aprendizaje hipotético- deductivo. 
- $\quad$ Relaciones entre los objetos: Capacidad para relacionarse con los objetos buscando causas y lógica. Comprende dos tipos de relación. Relaciones de causalidad, que es la capacidad del sujeto para la resolución de problemas utilizando objetos o utensilios junto con su representación, lo que facilitará la creación de relaciones entre las acciones, los objetos y las metas. Relaciones de lógica, referido al sistema jerárquico que permite organizar el mundo en categorías y establecer entre ellas relaciones interconceptuales. El lenguaje es importante para su desarrollo en el infante.

\section{Desarrollo de las habilidades protomentalistas.}

Los bebés comienzan la vida con interés y preferencia por la estimulación social. Son las voces y los rostros humanos, las formas más efectivas de captar su atención (Rochat, 2001).

Según Moore (2010) de los dos a los tres meses, los bebés son capaces de participar en interacciones sociales simples con otras personas mediante las cuales, pueden coordinar sus gestos, vocalizaciones y expresiones faciales con los otros. La estimulación social llega hacer un patrón confiable que juega un papel importante en este desarrollo. Sin embargo, otros cambios que son más producto de la madurez como la organización cerebral, la cognición y su complejidad también parecen ser importantes.

De los 4 a los 6 meses de edad, los bebés comienzan a participar en actividades conjuntas o compartidas con objetos como sus juguetes. Pueden participar en juegos simples de turnos con otras personas; además pueden conseguir dirigir la atención de los demás, pueden desarrollar emociones hacia los objetos basadas en las emociones que otros expresan; Pueden aprender nuevas formas de relacionarse con los objetos, imitando directamente a los demás. Este tipo de comportamientos indican que los bebés, se hacen cada vez más sensibles y comprensivos de los estados psicológicos de los demás, aunque al principio esta comprensión se manifiesta solo en situaciones en las que los bebés pueden compartir tales estados psicológicos con otros (Moll \& Tomasello, 2007).

A lo largo de los 6 meses de edad, los bebés ven acciones simples, pero con objetivos dirigidos como alcanzar un objeto, realizan acciones cada vez más complejas y secuenciales. A esta edad, los bebés también pueden diferenciar entre acciones accidentales e intencionales, y reconocer que solo seres animados (y no los objetos inanimados) poseen metas e intenciones. Otro aspecto importante de la cognición social consiste en reconocer el significado de los actos perceptivos y de las expresiones emocionales (Sommerville, 2010).

A partir de los 9 a 12 meses de edad, para Sommerville (2010) los bebés parecen comprender experiencias perceptivas simples y reconocer el valor de las diferentes expresiones emocionales. Por ejemplo, los bebés reconocen que los adultos, si miran un juguete con los ojos abiertos, tienen una experiencia de tipo perceptiva, pero este mismo no la tiene si mira el juguete con los ojos cerrados (Brooks \& Meltzoff, 2005). Asimismo, los bebés logran desarrollar la referencia social, debido a que pueden usar la expresión emocional de un experimentador o de sus padres para decidir si deben o no acercarse a un juguete nuevo (Baldwin \& Moses, 2001) o participar en una actividad novedosa (Adolph, Tamis, Ishak, Karasik \& Lobo, 2008). La capacidad de saber cómo las características personales influyen en el comportamiento también es un aspecto crítico de la cognición social.

Entre los 12 y los 15 meses, los bebés comienzan a comprender disposiciones y preferencias simples. Por ejemplo, en los bebés de esta edad esperan que un personaje continúe con una conducta o actividad previa cuando se los coloca en una novela. Además, los bebés entienden que las personas tienen preferencias y disposiciones personales, es decir logran 
comprender que a diferentes personas les pueden gustar cosas diferentes (Kuhlmeier, Wynn \& Blomm; Song, Baillargeon \& Fisher, citados por Sommerville, 2010).

En los primeros 18 a 24 meses, los bebés son capaces de reconocer que otros pueden experimentar estados psicológicos que son diferentes de los suyos, por ejemplo, pueden comprender que alguien no puede ver algo que ellos sí pueden ver o que alguien puede sentir algo que ellos no sienten. Al mismo tiempo, los bebés muestran una clara evidencia de autoconciencia, como reconocer su imagen en un espejo. Este desarrollo da lugar a profundos cambios en las relaciones sociales, comportamiento social y comportamiento cooperativo con los demás, estos comportamientos se hacen más eficaces (Warneken \& Tomasello, 2009). Paralelamente, los bebés se convierten en personas más autónomas, capaces y dispuestas a expresar y ejercer su independencia. Estos hallazgos revelan que el desarrollo de la cognición social en la infancia es progresivo e incluso se desarrolla antes de que el lenguaje se halla establecido (Herol \& Akhtar, 2008). Es decir, los bebés comienzan a mostrar un comportamiento empático y prosocial hacia los demás, a pesar de que aún no pueden usar el lenguaje para interactuar con otros o expresar su comprensión. Por lo tanto, Moore (2010) propone que es importante explorar sobre el desarrollo de la cognición social infantil para poder explicar que es lo que los bebés entienden de sí mismos y de los demás sin depender del lenguaje.

Sommerville (2010) señala que al final de los dos años de vida, los bebés son expertos en entender las intenciones, los objetivos básicos, las percepciones y expresiones emocionales, así como las preferencias y disposiciones simples de otras personas. Estas habilidades sociocognitivas son los bloques de construcción para aspectos más maduros de la cognición social, como la teoría de la mente (Astington \& Dack, 2008; Miller, 2010). Además, las capacidades sociocognitivas tempranas contribuyen al aprendizaje en una variedad de dominios, tales como el aprendizaje de idiomas, el aprendizaje imitativo, el aprendizaje causal y la comprensión representacional. A los niños de tres años saben que diferentes personas pueden querer, gustar y sentir cosas diferentes.

Entre las edades de 4 y 5 , los niños realmente comienzan a pensar en los pensamientos y sentimientos de los demás, y es allí, cuando surge la verdadera teoría de la mente (Peterson et al., 2012; Sussman, 2006; Wellman \& Liu, 2004). La teoría mental de los niños continúa desarrollándose después de los cinco años. Durante los próximos años, aprenderán a predecir lo que una persona piensa o siente acerca de lo que otra persona está pensando o sintiendo. Es así que los niños comienzan a comprender el lenguaje complejo como las mentiras, el sarcasmo y el lenguaje figurado que tienen como base la teoría de la mente (Wellman \& Liu, 2004).

Según, Astington y Edward (2010) las habilidades mentalistas o teoría de la mente se desarrollan gradualmente, con habilidades sociales intuitivas que aparecen en la infancia y luego se desarrolla la cognición social reflexiva durante los años de preescolar y niños de los primeros años de primaria. Algunos expertos manifiestan que el desarrollo de la teoría de la mente continúa durante toda la vida, ya que uno tiene más oportunidades de experimentar a las personas y su comportamiento (de Villiers \& de Villiers, 2014; Miller, 2012).

\section{Factores sociales e internos que desarrollan las habilidades mentalistas.}

Para Astington y Edward (2010) existen algunos factores en el entorno social e internos que influyen en el desarrollo típico de la teoría de la mente:

- Si las madres hablan sobres sus pensamientos, deseos y sentimientos, y proporcionan razones para corregir el mal comportamiento, lograran que los niños evidencien una conciencia más temprana de los estados mentales.

- El tener hermanos y/o hermanas, estimulan la conciencia de los estados mentales.

- El participar en juegos de roles o de simulación, estimulan el desarrollo de la teoría de la mente. 
- Tener experiencias de lectura de libros de cuentos.

- Hablar con otros sobre experiencias pasadas.

- Dentro de los factores internos del niño, que influyen en el desarrollo de las habilidades mentalistas, están:

- Habilidades del lenguaje, y habilidades cognitivas que controlan y regulan el comportamiento (conocidas como funciones ejecutivas).

\section{Funcionalidad de las habilidades protomentalistas.}

Desde la primera infancia, los niños aprenden las protohabilidades que necesitarán para desarrollar sus propias habilidades mentalistas más adelante. Westby y Robinson (2014) y de Villiers y de Villiers (2014) sugieren que las protohabilidades permiten a los infantes:

- Prestar atención a las personas e imitarlas.

- El reconocimiento de emociones en otras personas y utilizar palabras para expresarlas ("enojado", "feliz", "triste").

- Simular ser otra persona (como una vendedora, un médico o una profesora) cuando juegan.

- Comprender las causas y consecuencias de las emociones ("si arrojo mi juguete, mi mamá se enojará").

- Saber que son diferentes a las otras personas y que tienen diferentes gustos/disgustos de los demás.

- Saber que las personas actúan de acuerdo con lo que quieren.

Las investigaciones muestran que el desarrollo de la teoría de la mente tiene consecuencias para el funcionamiento social y el éxito escolar de los niños, sus maestros los califican como socialmente más competentes. Los niños con una teoría de la mente más desarrollada son mejores comunicadores y pueden resolver conflictos con sus amigos de manera más eficiente; su juego simulado es más complejo; impresionan ser más felices en la escuela y son más populares entre sus compañeros; y su trabajo escolar es más avanzado en algunos aspectos. Sin embargo, una teoría de la mente bien desarrollada también puede usarse de manera antisocial, como realizar burlas, conductas de acoso y mentiras (Astington \& Edward, 2010).

\section{Problemas en el desarrollo de las habilidades mentalistas.}

Kimbi (2014) y Lowry (2016) indican que el pobre desarrollo de las habilidades mentalistas lleva a los infantes a tener dificultades en su interacción y adaptación social, por ejemplo, existen muchas habilidades que se les hacen difíciles de realizar, tales como:

- Participar en juegos simbólicos donde personifiquen y jueguen al "como si".

- Mantener una conversación socialmente aceptable.

- Entender por qué la gente dice y hace lo que hace.

- Narrar una historia de forma espontánea, sin yuxtaposiciones.

- Entender los puntos de vista de los personajes en los libros de cuentos.

- Hacer amigos en la escuela o en su comunidad.

\section{Importancia de la evaluación de las habilidades protomentalistas.}

Westby y Robinson (2014) manifiestan que en la actualidad la existencia de herramientas estandarizadas disponibles para evaluar las dimensiones de las habilidades mentalistas o teoría de la mente por separado son escasas, esta situación se extrapola con mayor razón a la evaluación de las habilidades protomentalistas que son aún menos estudiadas desde un enfoque más psicométrico. Es así como, ante esta problemática, proponen que para evaluar las habilidades mentalistas o protomentalistas debemos observar el curso del patrón evolutivo que siguen estas 
habilidades para comprenderlas y de este modo poder iniciar el conocimiento de su estado de desarrollo en los infantes.

Sommerville (2010) señala que, para evaluar la cognición social temprana, los investigadores deben confiar en métodos no verbales, novedosos e innovadores, debido a que los bebés no pueden realizar tareas basadas en el lenguaje por su escaso desarrollo. Los métodos para la evaluación se dan en contextos naturales o experimentales, donde se aprovechan para la evaluación del comportamiento social de los bebés, es así como las respuestas visuales a eventos sociales simples han proporcionado una gran cantidad de información con respecto a la cognición social temprana. Sin embargo, como los hallazgos de estos métodos a menudo están abiertos a múltiples interpretaciones, aun se necesitan métodos convergentes para obtener una imagen precisa y cuidadosamente controlada de la cognición social en la infancia y así reducir significativamente el número de posibles interpretaciones alternativas de los resultados de la investigación.

\section{Objetivo de la investigación.}

El objetivo de la presente investigación fue construir un instrumento que se ajuste al modelo teórico de la existencia de las habilidades previas a la mentalización o teoría de la mente y que estas se ajusten a las habilidades del constructo habilidades protomentalistas, identificadas y reconocidas en estudios anteriores. A su vez, nos propusimos recolectar evidencia de validez basada en el contenido de test a partir del análisis respecto a las valoraciones de un grupo de jueces expertos de la versión piloto de la escala para la evaluación del desarrollo de Habilidades Protomentalistas (PROTO - HM) en menores de 3 años de Lima, Perú, haciendo uso del coeficiente V de Aiken (Aiken, 1985; Merino \& Livia, 2009).

\section{Método}

\section{Participantes.}

La escala PROTO - HM fue enviada a un grupo de jueces quienes valoraron el contenido de los ítems del instrumento psicométrico. Los jueces expertos fueron seleccionados de acuerdo con su área de especialización como conocimiento basto en el desarrollo evolutivo de la primera infancia y experiencia psicoeducativa en intervención temprana. Estos cumplieron el rol de jueces evaluando la claridad, relevancia y coherencia de los 80 ítems que se propusieron originalmente. Para este fin, se logró la participación de 12 jueces residentes en Estados Unidos (1), Chile (1) y Perú (10). Ocho expertos se dedican a la docencia e investigación, teniendo publicaciones en revistas científicas sobre desarrollo infantil $\mathrm{y}$, los otros cuatro jueces son profesionales dedicados a la intervención psicoeducativa de infantes con y sin desarrollo neurotípico.

\section{Instrumento.}

Utilizando como referencia los instrumentos para la detección de habilidades mentalistas en la primera infancia de Sáiz y Guijo (2011) y la dimensión social del inventario I.D.E.A. de Riviére y Martos (1997), se elaboró un instrumento adaptado a la realidad de los infantes menores de 3 años de nuestro contexto. La escala fue diseñada como un producto de la operacionalización previa de las variables habilidades protomentalistas de interacción con los objetos y de interacción social, de allí que se obtuvo diversos indicadores que hacían factible su observación y medida.

Las habilidades protomentalistas son precursoras de las actividades mentalistas, que se desarrollan antes de los cuatro años y que son mecanismos que ayudan al bebé humano a acceder al espacio mental compartido. Las habilidades de interacción con las personas son capacidades que se producen por mediación social y que están conformadas por las relaciones triangulares, la intersubjetividad, el simbolismo y la referencia social. Por otro lado, las habilidades de interacción con los objetos son capacidades que surgen gracias a la mediación instrumental y que posibilitan 
el desarrollo de la representación mental y están conformadas por la permanencia del objeto, la relación entre los objetos (causalidad y lógica), la inteligencia práctica y la resolución de problemas con objetos, estas variables se midieron a partir de una selección final de aquellas que se consideró conveniente para cada una de las dimensiones mencionadas.

Posteriormente, se construyó una matriz instrumental o de operacionalización de variables para descomponer en dimensiones e indicadores las variables de estudio y obtener las posibilidades de elaboración de ítems en relación con las dimensiones de las habilidades protomentales. Seguidamente, se elaboró una versión piloto del instrumento. Esta escala contenía, tanto preguntas para los padres, observaciones conductuales de los infantes como tareas para ejecutar por los niños. Asimismo, se procuró brindar una herramienta con material de evaluación amigable y con mayor atractivo, que permitiera recopilar información de forma valida e interactiva, congruente con las capacidades cognitivas, edad cronológica y desarrollo social de los infantes.

La versión original presentó 80 reactivos diseñados en razón proporcional a las dimensiones que se evaluaron. La dimensión habilidades de interacción con las personas estuvo compuesta por 61 ítems y la dimensión habilidades de interacción con los objetos por 19 ítems. Asimismo, la prueba tiene ítems que presentan tres formas de aplicación que podrían darse de forma conjunta o separada: Observación (O), Tarea Estructurada (TE) aplicada al infante y Entrevista informacional (E) de los padres o apoderado del niño. Estas respuestas fueron categorizadas en puntuaciones generales de 2 puntos si realizaba la habilidad siempre (S), 1 punto si lo hacía A veces (AV) y finalmente 0 puntos si no lo realizaba $(\mathrm{N})$. Es importe mencionar que cada reactivo tiene una forma cualitativa y especifica de calificar, pero todas se basan en las categorías cuantitativas mencionadas. La aplicación es individual y toma aproximadamente 30 minutos.

\section{Procedimiento.}

Se utilizó un diseño de investigación instrumental (Ato, López \& Benavente, 2013). Para lograr el análisis de contenido de la escala, se solicitó la valoración de jueces expertos u opinión informada de personas con autoridad (Escobar-Pérez \& Cuervo- Martínez, 2008) para evaluar el grado de claridad, representación y relevancia del dominio que se buscaba medir, cada juez recibió una copia del instrumento original con 80 ítems y el protocolo de valoración, que incluía el objetivo de estudio, las instrucciones, con sus respectivas normas de calificación, y el acuerdo de anonimidad.

Se tuvo en consideración el carácter voluntario de la participación de los jueces. En un primer momento, se elaboró una lista de 15 profesionales expertos en el área de desarrollo evolutivo infantil y en intervención temprana con la finalidad invitarlos como jueces expertos. La invitación se remitió a través de correos electrónicos personales y/o institucionales, esto debido a la variedad de lugares donde vivían los jueces y el poco tiempo para realizar un contacto personal. Los lugares de residencia de los jueces fueron Estados Unidos, Chile y Perú. Finalmente, 12 expertos aceptaron participar en el estudio; dos invitados declinaron a participar por razones de tiempo y un profesional no dio respuesta a la solicitud. La presentación de la escala y las instrucciones a los jueces/expertos fueron las siguientes:

«Usted ha sido elegido como experto para emitir su opinión sobre el contenido de los 80 ítems de esta escala. Su experiencia es muy valiosa para formarnos una opinión consensual sobre la calidad de los ítems en este proceso de construcción del instrumento. Permítanos asegurarle que su participación será mantenida de manera confidencial, y es voluntaria. Esta prueba está diseñada para valorar el desarrollo madurativo de las habilidades proto o pre mentalistas del niño durante los tres primeros años de vida. Estas habilidades previas ayudan al infante a interaccionar con las personas y en su adaptación social. Los participantes serán infantes de 1 hasta 47 meses de edad, así como sus cuidadores, en especial sus madres». 
«De los 80 ítems, 61 de ellos corresponden a 4 distintas dimensiones de las habilidades protomentalistas de interacción con las personas: 1) Relaciones Triangulares, 2) Intersubjetividad, 3) Simbolización, y 4) Referencia Social». La tabla 1 contiene los 61 ítems originales del instrumento.

\section{Tabla 1.}

Lista de los 61 ítems de la Escala de Habilidades Protomentalistas de interacción con las personas.

Dimensión I: Relaciones Triangulares

Contacto ocular

1.Mantiene contacto ocular espontáneo con las personas cuando explora el ambiente.

2. Mantiene contacto ocular ante orden "mírame" o al llamarlo por su nombre.

\section{Atención}

3. Mantiene la atención sostenida mientras escucha que el adulto le propone hacer un juego o actividad.

4. Mantiene la atención cuando ejecuta una tarea o juego propuesto por el adulto.

5. Mantiene la atención sostenida cuando ejecuta una tarea o juego de forma espontánea.

Pautas de atención conjunta

6. Toma parte en juegos sociales como el "Aquí está el Coco".

7. Se observa que acompaña al adulto en su estado emocional.

8. Se observa que acompaña niños/as de su edad o grupo en el estado emocional.

9. Sigue las pautas de atención compartida.

Uso del proto imperativo

10. Incorpora de forma intencional a un adulto como un agente o instrumento para alcanzar algún fin, en concreto un objeto deseado.

Uso del proto declarativo

11. Realiza esfuerzos pre verbales por atraer la atención del adulto sobre un suceso u objeto haciendo uso de un objeto para captar la atención del adulto.

Dimensión II: Intersubjetividad

Intersubjetividad Primaria

12. Imita las acciones conocidas que realiza un modelo.

13. Realiza protoconversaciones, es decir intercambia con un adulto sonidos con intención de comunicarse durante sus actividades de vida diaria.

14.Sincroniza interactivamente sus conductas, realizando movimientos ligados a la voz humana.

15. Relaciones cara a cara: el bebé muestra diferentes expresiones faciales.

Intersubjetividad Secundaria

Actos Práxicos

16.Señala con el dedo el objeto que le interesa.

17.Muestra cosas de su interés a las personas que le rodean.

18.Da cosas que se le piden.

19. Ofrece cosas a las demás personas.

20. Extiende la mano para obtener algo de su interés.

Interpersonal 
21. Sonríe frente a situaciones graciosas o novedosas.

22. Articula sonidos o palabras para comunicarse.

23.Mira a la cara de las personas cuando se comunica.

24. Busca al adulto para comunicarle lo que sucede con un objeto, persona o situación, en el momento presente.

25.Busca al adulto para comunicarle algo a cerca de un objeto, persona o una situación que NO está presente sino en el recuerdo o representada en la mente del niño/a.

Dimensión III: Simbólica

Imitación

26. Imita sonidos o palabras que escucha de otras personas.

27. Imita acciones motoras que observó en otras personas.

Juego Simbólico

28. Juega representando de forma ficticia ("como si") acciones cotidianas de su vida diaria, haciendo uso de su cuerpo.

29. Juega repartiendo roles ("como si") a los muñecos o personas, quienes esperan o reciben la acción planteada por el niño/a.

30. Juega usando los objetos reales y en tamaño natural o estos representados como juguetes.

31. Al jugar reemplaza un objeto real y conocido por uno indefinido simulando acciones cotidianas.

32. Sus juegos son actividades cortas, simples y aisladas (no secuenciadas) para ello utiliza dos o más objetos o agentes.

33. Juega con objetos o muñecos y les hace representar actividades o acciones de la vida cotidiana.

34. En sus juegos hace participar activamente a sus muñecos en las situaciones que representa, como si estos tuvieran vida.

35. Al jugar realiza acciones con sus muñecos como protagonistas, donde el niño/a efectúa verbalizaciones como si fuese el muñeco el que hablase.

36. Al jugar utiliza objetos reales con función definida y les reemplaza la función para las que fueron creadas simulando acciones cotidianas.

37. Sus juegos representan secuencias verdaderas, más de dos actividades cortas en las que se incluyen dos o más acciones simbólicas se observa que la secuencia evoluciona sin planeación previa.

38. Antes de comenzar a jugar busca lo juguetes u objetos que necesitará para su juego y los lleva a un lugar determinado, los acomoda y realiza la acción lúdica.

Dimensión IV: Habilidades de Referencia Social

Respuesta a la manifestación de emociones de otras personas.

39. Cuando un adulto le muestra alegría, responde con la misma emoción.

40. Cuando un adulto le muestra tristeza, responde con la misma emoción.

41. Cuando un adulto le muestra enfado, responde con la misma emoción.

42. Cuando un adulto le muestra sorpresa, responde con la misma emoción.

43. Cuando un niño le muestra alegría, responde con la misma emoción.

44. Cuando un niño le muestra tristeza, responde con la misma emoción.

45. Cuando un niño le muestra enfado, responde con la misma emoción

46. Cuando un niño le muestra sorpresa, responde con la misma emoción.

Manifestación de emociones frente a las personas

47. Le sonríe a un adulto conocido cuando se aproxima a él o ella.

48. Le sonríe a un adulto desconocido cuando se aproxima a él o ella.

49. Cuando está con adultos que no conoce, manifiesta susto y/o desconfianza.

50. Realiza acciones o tareas que le pide un adulto que conoce. 
51. Cuando un adulto le presenta algo novedoso (tarea, situación u objeto), sacude los brazos o agita alguna parte de su cuerpo como señal de alegría.

52. Cuando un adulto que conoce se va, manifiesta tristeza en su rostro.

Identificación de emociones

53. Diferencia cual es un objeto y cual es una persona.

54. Identifica a las personas que muestran alegría.

55. Identifica a las personas que muestran tristeza.

56. Identifica a las personas que muestran enfado.

57. Identifica a las personas muestran sorpresa.

58. Identifica sus expresiones de alegría.

59. Identifica sus expresiones de tristeza.

60. Identifica sus expresiones de enfado.

61. Identifica sus expresiones de sorpresa.

«Y los otros 19 ítems corresponden a 3 dimensiones de las habilidades protomentalistas de interacción con los objetos: 1) Permanencia del objeto, 2) Inteligencia practica y resolución de problemas con objetos, y 3) Relaciones entre objetos (causalidad y lógica). Como experto, usted deberá calificar los ítems en un rango del 1 (nada) al 5 (completamente), en 3 aspectos de análisis: Claridad, Relevancia y Representatividad. La Claridad designa si el ítem es entendible, claro y comprensible; la Relevancia se refiere a la relación del ítem con el constructo de estudio; y la Representatividad se refiere así el ítem es representativo e importante para la medición del constructo habilidades protomentalistas». La tabla 2 contiene los 19 ítems originales del instrumento.

\section{Tabla 2.}

Lista de los 19 items de la Escala de Habilidades Protomentalistas de interacción con los objetos.

Dimensión I: Permanencia del objeto.

1. Sigue visualmente un objeto en movimiento.

2. Mira atentamente el punto en el que ha desaparecido un objeto, pero aún no lo busca cuando desaparece.

3. Busca el objeto parcialmente oculto.

4. Busca el objeto totalmente oculto que acaba de desaparecer.

5. Descubre el mismo objeto en los distintos sitios que se va ocultando. Aún no es capaz de tener en cuenta los desplazamientos no visibles.

6. Busca el objeto en los lugares donde se escondieron por medio de desplazamientos invisibles.

Dimensión II: Inteligencia practica y resolución de problemas con objetos

Estrategias medio - fin:

7. Coordina la prensión y la visión al coger los objetos pequeños.

8. Manifiesta intención por alcanzar objetos fuera de su alcance, pero lo hace al azar de forma lenta y no sistemática y sin usar soportes o herramientas como medio para alcanzar objetos.

9. Realiza tareas simples a través de acciones intencionadas, pero no muy coordinadas y eficientes, se inicia en el uso de soportes o herramientas.

10. Resuelve tareas sencillas que le permiten obtener el objeto que desea a través de conductas intencionadas y coordinadas, utilizando herramientas de forma eficiente.

11. Resuelve tareas diferenciando entre las herramientas o medios que tiene que utilizar para conseguir algo.

12. Utiliza estrategias de anticipación de por lo menos 3 pasos para lograr el objetivo, es decir cuando quiere obtener algo que desea, realiza acciones que evitan o superan los obstáculos presentes en la situación. 
13. Utiliza estrategias de anticipación referente a objetos con corrección de acciones para evitar errores y automonitorea su conducta.

14. Utiliza estrategias de anticipación referente a personas, es decir asocia la aparición y desaparición de personas conocidas con hechos cotidianos.

15. Inicia estrategias de planificación ("crea una representación adelantada de la acción") solucionando problemas por medio de su capacidad de análisis que lo lleva a utilizar medios para lograr su objetivo. Estos medios parten de la representación que hace en su mente y no solo de la acción directa.

Dimensión III: Relaciones entre los objetos (causalidad y lógica).

16. Realiza cualquier acción que indique que desea que se siga repitiendo "algo interesante".

17. Realiza acciones coordinadas y dirigidas para que se siga repitiendo "algo interesante" relacionando herramienta y acto.

18. Realiza de forma autónoma acciones coordinadas como consecuencia de un estímulo o solicitud.

19. Manifiesta curiosidad por encontrar la fuente del estímulo, buscando quien realiza el movimiento del objeto o qué produce un sonido.

\section{Análisis de datos}

Para Sánchez y Echeverry (2004) el obtener la validación psicométrica de un instrumento involucra que este evidencie características básicas que son requeridas para que pueda utilizarse en una población específica de manera que se asegure que los resultados de sus mediciones son éticas, precisas, exactas y estables.

La evidencia de validez basada en el contenido de las escalas de habilidades protomentalistas, considerada una de las cinco fuentes de evidencias de validez, fue recolectada mediante el criterio de 12 jueces expertos cualificados (Skjong \& Wentworht, 2001) en los temas de desarrollo infantil, quienes dieron información, evidencia, juicio y evaluaron la claridad, relevancia y representatividad de los ítems respecto a las dimensiones que miden. Para obtener un índice cuantitativo de cada uno de estos aspectos, se empleó el coeficiente V de Aiken (Aiken, 1985) que permite evaluar el grado de acuerdo en la opinión de expertos sobre la validez de contenido de un instrumento, la relevancia de un ítem o la calidad de la redacción. Este coeficiente varía entre 0.00 y 1.00 , donde más cercano a la unidad indica una mejorar calidad del ítem respecto al aspecto evaluado (claridad, relevancia o representatividad), se estableció como valor crítico una $\mathrm{V}=.50$. Asimismo, se calcularon los intervalos de confianza (Penfield \& Giacobbi, 2004), a un nivel del 95\% donde, aquellos casos cuyo límite inferior fue mayor a .50, se consideraron aceptables. Se utilizó el programa desarrollado por Merino y Livia (2009) para calcular la V de Aiken y los intervalos de confianza para el coeficiente V.

\section{Resultados}

A continuación, se presentan los resultados del análisis cuantitativo de las valoraciones de los jueces respecto al contenido de los ítems sobre la claridad, la relevancia y la representatividad de mismos, a partir del coeficiente V de Aiken de la escala PROTO - HM para cada dimensión.

En relación con los ítems de la escala PROTO - HM de interacción con las personas (tabla 3), todos presentaron valores superiores a .80 en los tres aspectos considerados en la evaluación del contenido de los ítems. Esto indica que, el contenido de los ítems se ajusta a las dimensiones a las que pertenecen, y en general, al constructo que miden. Estos resultados brindan evidencias de contenido a las inferencias que se puedan realizar a partir de los puntajes que se obtengan en esta escala. Complementariamente, los límites inferiores del intervalo de confianza fueron mayores a .50 en todos los casos, indicativo del buen funcionamiento de los ítems. 
Tabla 3.

Intervalos de confianza en el coeficiente V (95\%) de la escala PROTO HM de interacción con las personas.

\begin{tabular}{|c|c|c|c|c|c|c|c|c|c|}
\hline \multirow[b]{3}{*}{ Ítem } & \multicolumn{3}{|c|}{ Claridad } & \multicolumn{3}{|c|}{ Relevancia } & \multicolumn{3}{|c|}{ Representatividad } \\
\hline & \multirow[b]{2}{*}{$V$} & \multicolumn{2}{|c|}{$95 \% \mathrm{IC}$} & \multirow[b]{2}{*}{$V$} & \multicolumn{2}{|c|}{$95 \% \mathrm{IC}$} & \multirow[b]{2}{*}{$V$} & \multicolumn{2}{|c|}{$95 \%$ IC } \\
\hline & & $L I$ & $L S$ & & $L I$ & $L S$ & & $L I$ & $L S$ \\
\hline \multicolumn{10}{|c|}{ Dimensión I: Relaciones triangulares } \\
\hline \multicolumn{10}{|c|}{ Contacto ocular } \\
\hline Ítem_1 & .967 & .885 & .991 & 1.00 & .940 & 1.00 & 1.00 & .940 & 1.00 \\
\hline Ítem_2 & .933 & .842 & .974 & 1.00 & .940 & 1.00 & 1.00 & .940 & 1.00 \\
\hline \multicolumn{10}{|l|}{ Atención } \\
\hline Ítem_3 & .867 & .758 & .930 & 1.00 & .940 & 1.00 & 1.00 & .940 & 1.00 \\
\hline Ítem_4 & .800 & .682 & .882 & .933 & .842 & .974 & .933 & .842 & .974 \\
\hline Ítem_5 & .867 & .758 & .930 & .933 & .842 & .974 & .967 & .885 & .991 \\
\hline \multicolumn{10}{|c|}{ Pautas de atención conjunta } \\
\hline Ítem_6 & .900 & .799 & .953 & 1.00 & .940 & 1.00 & 1.00 & .940 & 1.00 \\
\hline Ítem_7 & .900 & .799 & .953 & .967 & .885 & .991 & .967 & .885 & .991 \\
\hline Ítem_8 & .933 & .842 & .974 & .933 & .842 & .974 & .967 & .885 & .991 \\
\hline Ítem_9 & .933 & .842 & .974 & .900 & .799 & .953 & .900 & .799 & .953 \\
\hline \multicolumn{10}{|c|}{ Uso del proto imperativo } \\
\hline Ítem_10 & .933 & .842 & .974 & .967 & .885 & .991 & .967 & .885 & .991 \\
\hline \multicolumn{10}{|c|}{ Uso del proto declarativo } \\
\hline Ítem_11 & .933 & .842 & .974 & .967 & .885 & .991 & 1.00 & .940 & 1.00 \\
\hline \multicolumn{10}{|c|}{ Dimensión II: Intersubjetividad } \\
\hline \multicolumn{10}{|c|}{ Intersubjetividad primaria } \\
\hline Ítem_12 & .833 & .720 & .907 & .900 & .799 & .953 & .900 & .799 & .953 \\
\hline İtem_13 & 967 & .885 & .991 & 1.00 & .940 & 1.00 & 1.00 & .940 & 1.00 \\
\hline Ítem_14 & .900 & .799 & .953 & .933 & .842 & .974 & .933 & .842 & .974 \\
\hline Ítem_15 & .833 & .720 & .907 & .967 & .885 & .991 & .967 & .885 & .991 \\
\hline \multicolumn{10}{|c|}{ Intersubjetividad secundaria } \\
\hline Ítem_16 & .967 & .885 & .991 & 1.00 & .940 & 1.00 & 1.00 & .940 & 1.00 \\
\hline Ítem_17 & .933 & .842 & .974 & 1.00 & .940 & 1.00 & 1.00 & .940 & 1.00 \\
\hline Ítem_18 & .933 & .842 & .974 & 1.00 & .940 & 1.00 & 1.00 & .940 & 1.00 \\
\hline İtem_19 & 967 & .885 & .991 & 1.00 & .940 & 1.00 & 1.00 & .940 & 1.00 \\
\hline Ítem_20 & .933 & .842 & .974 & .967 & .885 & .991 & .967 & .885 & .991 \\
\hline Ítem_21 & .967 & .885 & .991 & .967 & .885 & .991 & .967 & .885 & .991 \\
\hline Ítem_22 & 967 & .885 & .991 & .967 & .885 & .991 & .967 & .885 & .991 \\
\hline Ítem_23 & 967 & .885 & .991 & .967 & .885 & .991 & .967 & .885 & .991 \\
\hline Ítem_24 & .933 & .842 & .974 & 1.00 & .940 & 1.00 & 1.00 & .940 & 1.00 \\
\hline Ítem_25 & .967 & .885 & .991 & .933 & .842 & .974 & .933 & .842 & .974 \\
\hline \multirow{2}{*}{\multicolumn{10}{|c|}{$\begin{array}{l}\text { Dimensión III: Simbólica } \\
\text { Imitación }\end{array}$}} \\
\hline & & & & & & & & & \\
\hline $\begin{array}{l}\text { Item_26 } \\
\text { Ítem_27 }\end{array}$ & $\begin{array}{l}.96 / \\
.967\end{array}$ & $\begin{array}{l}.885 \\
885\end{array}$ & $\begin{array}{r}991 \\
991\end{array}$ & $\begin{array}{l}1.00 \\
100\end{array}$ & $\begin{array}{r}.940 \\
940\end{array}$ & 1.00 & 1.00 & .940 & $\begin{array}{l}1.00 \\
100\end{array}$ \\
\hline Juego $\sin$ & .901 & . & .991 & 1.00 & .940 & 1.00 & 1.00 & .940 & 1.00 \\
\hline Ítem_28 & .967 & .885 & .991 & 1.00 & .940 & 1.00 & 1.00 & .940 & 1.00 \\
\hline Ítem_29 & .933 & .842 & .974 & .933 & .842 & .974 & .933 & .842 & .974 \\
\hline Ítem_30 & .933 & .842 & .974 & .933 & .842 & .974 & .933 & .842 & .974 \\
\hline Ítem_31 & .933 & .842 & .974 & .933 & .842 & .974 & .933 & .842 & .974 \\
\hline Ítem_32 & .900 & .799 & .953 & .933 & .842 & .974 & .933 & .842 & .974 \\
\hline Ítem_33 & .967 & .885 & 991 & .933 & .842 & .974 & .933 & .842 & .974 \\
\hline Ítem_34 & .900 & .799 & .953 & .933 & .842 & .974 & .933 & .842 & .974 \\
\hline Ítem_35 & .933 & .842 & .974 & .933 & .842 & .974 & .933 & .842 & .974 \\
\hline
\end{tabular}




\begin{tabular}{|c|c|c|c|c|c|c|c|c|c|}
\hline Ítem_36 & .800 & .682 & .882 & .933 & .842 & .974 & .933 & .842 & .974 \\
\hline Ítem_37 & .867 & .758 & .930 & .933 & .842 & .974 & .933 & .842 & .974 \\
\hline Ítem_38 & .933 & .842 & .974 & .867 & .758 & .930 & .867 & .758 & .930 \\
\hline \multirow{2}{*}{\multicolumn{10}{|c|}{$\begin{array}{l}\text { Dimensión IV: Habilidades de referencia social } \\
\text { Expresión de emociones }\end{array}$}} \\
\hline & & & & & & & & & \\
\hline $\begin{array}{l}\text { Item_39 } \\
\text { Ítem_40 }\end{array}$ & $\begin{array}{r}933 \\
933\end{array}$ & $\begin{array}{l}.842 \\
842\end{array}$ & $\begin{array}{r}.914 \\
974\end{array}$ & $\begin{array}{l}.933 \\
933\end{array}$ & $\begin{array}{r}.842 \\
842\end{array}$ & $\begin{array}{r}.914 \\
074\end{array}$ & $\begin{array}{r}.901 \\
933\end{array}$ & $\begin{array}{l}.885 \\
842\end{array}$ & $\begin{array}{r}991 \\
974\end{array}$ \\
\hline Ítem_41 41 & 933 & 842 & 974 & 933 & 842 & 974 & .933 & 842 & .974 \\
\hline Ítem_42 & .933 & .842 & .974 & .933 & .842 & .974 & .933 & .842 & .974 \\
\hline Ítem_43 & .933 & .842 & .974 & .933 & .842 & .974 & .933 & .842 & .974 \\
\hline Ítem_44 & 933 & .842 & .974 & .933 & .842 & .974 & .933 & .842 & .974 \\
\hline Ítem_45 & .933 & .842 & .974 & .933 & .842 & .974 & .933 & .842 & .974 \\
\hline Ítem_46 & .933 & .842 & .974 & .933 & .842 & .974 & .933 & .842 & .974 \\
\hline \multicolumn{10}{|c|}{ Manifestación de emociones } \\
\hline Ítem_47 & .967 & .885 & .991 & .967 & .885 & .991 & .967 & .885 & .991 \\
\hline Ítem_48 & .967 & .885 & .991 & .967 & .885 & .991 & .967 & .885 & .991 \\
\hline Ítem_49 & .967 & .885 & .991 & .967 & .885 & .991 & .967 & .885 & 991 \\
\hline Ítem_50 & .833 & .720 & .907 & .900 & .799 & .953 & .900 & .799 & .953 \\
\hline Ítem_51 & 967 & .885 & .991 & .967 & .885 & .991 & .967 & .885 & 991 \\
\hline Ítem_52 & .967 & .885 & .991 & .967 & .885 & .991 & .967 & .885 & 991 \\
\hline Ítem_53 & .900 & .799 & .953 & .833 & .720 & .907 & .833 & .720 & 907 \\
\hline \multicolumn{10}{|c|}{ Reconocimiento de emociones } \\
\hline Ítem_54 & .900 & .799 & .953 & .967 & .885 & .991 & .967 & .885 & .991 \\
\hline Ítem_55 & .900 & .799 & .953 & .967 & .885 & .991 & .967 & .885 & .991 \\
\hline Ítem_56 & .900 & .799 & .953 & .967 & .885 & .991 & .967 & .885 & 991 \\
\hline Ítem_57 & .900 & .799 & .953 & .967 & .885 & .991 & .967 & .885 & .991 \\
\hline Ítem_58 & .900 & .799 & .953 & .967 & .885 & .991 & .967 & .885 & .991 \\
\hline Ítem_59 & .900 & .799 & .953 & .967 & .885 & .991 & .967 & .885 & 991 \\
\hline Ítem_60 & .900 & .799 & .953 & .967 & .885 & .991 & .967 & .885 & .991 \\
\hline Ítem_61 & .900 & .799 & .953 & .967 & .885 & .991 & .967 & .885 & .991 \\
\hline
\end{tabular}

Nota. V = Coeficiente V de Aiken; IC = Intervalo de confianza; LI = Límite inferior; LS = Límite superior

En relación con las dimensiones y subdimensiones de la escala PROTO - HM de interacción con las personas (tabla 4), estas obtuvieron coeficientes V de Aiken por encima de .90, con excepción de los subdimensiones Atención e Intersubjetividad primaria, ambas respecto a la claridad de sus ítems, no obstante, estos valores fueron superiores a .80, considerándose un nivel adecuado. Los mejores índices se obtuvieron en la relevancia y representatividad de las dimensiones respecto al constructo que miden. Por tanto, a nivel de las variables que componen la escala de interacción con las personas, estas cuentan con adecuados niveles de evidencia de validez basada en el contenido de los ítems que las componen. 
Tabla 4.

Coeficientes $V$ de Aiken promedio para las variables de la Escala PROTO HM de Interacción con las personas.

\begin{tabular}{|c|c|c|c|}
\hline Variable & Claridad & Relevancia & Representatividad \\
\hline Dimensión I: Relaciones triangulares & .906 & .964 & .973 \\
\hline Contacto ocular & .950 & 1.00 & 1.00 \\
\hline Atención & .845 & .955 & .967 \\
\hline Pautas de atención conjunta & .917 & .950 & .959 \\
\hline Uso del proto imperativo & .933 & .967 & .967 \\
\hline Uso del proto declarativo & .933 & .967 & 1.00 \\
\hline Dimensión II: Intersubjetividad & .933 & .972 & .972 \\
\hline Intersubjetividad primaria & .883 & .950 & .950 \\
\hline Intersubjetividad secundaria & .953 & .980 & .980 \\
\hline Dimensión III: Simbólica & .923 & .943 & .943 \\
\hline Imitación & 967 & 1.00 & 1.00 \\
\hline Juego simbólico & .915 & .933 & .933 \\
\hline $\begin{array}{l}\text { Dimensión IV: Habilidades de } \\
\text { referencia social }\end{array}$ & .923 & .946 & .948 \\
\hline Expresión de emociones & .933 & .933 & .937 \\
\hline Manifestación de emociones & .938 & .938 & .938 \\
\hline Reconocimiento de emociones & .900 & .967 & 967 \\
\hline
\end{tabular}

Los ítems de la escala PROTO - HM de interacción con los objetos (tabla 5), presentaron coeficientes V de Aiken por encima de .80 en claridad, relevancia y representatividad, considerándose este nivel adecuado. Únicamente el ítem 13 de la subdimensión Estrategias de anticipación y planificación presentó problemas respecto a su claridad, obteniendo un coeficiente $\mathrm{V}=.733$, no obstante, se encuentra aún en los límites permitidos (superior a .70), por lo que, se consideró mantenerlo en la escala previa modificación para mejorar su redacción y comprensión. Adicionalmente, los límites inferiores del intervalo de confianza de los coeficientes $\mathrm{V}$ de Aiken fueron superiores a .50 en todos los ítems, indicando el buen funcionamiento de los ítems.

Tabla 5.

Intervalos de confianza en el coeficiente V(95\%) de la Escala PROTO HM de Interacción con los objetos.

\begin{tabular}{|c|c|c|c|c|c|c|c|c|c|}
\hline & \multicolumn{3}{|c|}{ Claridad } & \multicolumn{3}{|c|}{ Relevancia } & \multicolumn{3}{|c|}{ Representatividad } \\
\hline & \multirow[b]{2}{*}{$V$} & \multicolumn{2}{|c|}{$95 \%$ IC } & \multicolumn{3}{|c|}{$95 \%$ IC } & \multirow[b]{2}{*}{$V$} & \multicolumn{2}{|c|}{$95 \%$ IC } \\
\hline & & $L I$ & $L S$ & $V$ & $L I$ & $L S$ & & $L I$ & $L S$ \\
\hline \multicolumn{10}{|c|}{ Dimensión I: Permanencia del objeto } \\
\hline Ítem_1 & .933 & .842 & .974 & 1.00 & .940 & 1.00 & 1.00 & .940 & 1.00 \\
\hline Ítem_2 & .917 & .818 & .963 & .983 & .913 & .997 & 1.00 & .940 & 1.00 \\
\hline Ítem_3 & .950 & .863 & .983 & .967 & .885 & 991 & 1.00 & .940 & 1.00 \\
\hline Ítem_4 & .950 & .863 & .983 & .983 & .913 & 997 & 1.00 & .940 & 1.00 \\
\hline Ítem_5 & .933 & .842 & .974 & .983 & .913 & 997 & 1.00 & .940 & 1.00 \\
\hline Ítem_6 & .933 & .842 & .974 & .983 & .913 & 997 & 1.00 & .940 & 1.00 \\
\hline \multicolumn{10}{|c|}{ Dimensión II: Inteligencia práctica } \\
\hline \multicolumn{10}{|c|}{ Estrategias medio-fin } \\
\hline Ítem_7 & .833 & .720 & .907 & .900 & .799 & .953 & .900 & .799 & .953 \\
\hline Ítem_8 & .900 & .799 & .953 & .933 & .842 & .974 & .933 & .842 & .974 \\
\hline Ítem_9 & .900 & .799 & .953 & .967 & .885 & 991 & .967 & .885 & .991 \\
\hline Ítem_10 & .933 & .842 & .974 & .967 & .885 & 991 & 1.00 & .940 & 1.00 \\
\hline
\end{tabular}




\begin{tabular}{lccccccccc} 
Ítem_11 & .933 & .842 & .974 & 1.00 & .940 & 1.00 & 1.00 & .940 & 1.00 \\
Estrategias de anticipación & y planificación & & & & & & \\
Ítem_12 & .933 & .842 & .974 & .967 & .885 & .991 & 1.00 & .940 & 1.00 \\
Ítem_13 & .733 & .611 & .829 & .900 & .799 & .953 & .900 & .799 & .953 \\
Ítem_14 & .867 & .758 & .930 & .933 & .842 & .974 & .933 & .842 & .974 \\
Ítem_15 & .933 & .842 & .974 & .933 & .842 & .974 & .933 & .842 & .974 \\
Dimensión III: Relaciones entre los objetos & & & & & & \\
Ítem_16 & .920 & .824 & .966 & .960 & .877 & .988 & .960 & .877 & .988 \\
Ítem_17 & .880 & .774 & .940 & .960 & .877 & .988 & .960 & .877 & .988 \\
Ítem_18 & .920 & .824 & .966 & .960 & .877 & .988 & .960 & .877 & .988 \\
Ítem_19 & .920 & .824 & .966 & .960 & .877 & .988 & .960 & .877 & .988 \\
\hline
\end{tabular}

Nota. V = Coeficiente V de Aiken; IC = Intervalo de confianza; LI = Límite inferior; LS = Límite superior

Considerando las dimensiones y subdimensiones de la escala PROTO - HM de interacción con los objetos (tabla 6), estas cuentan con coeficientes V de Aiken adecuados, en todos los casos superiores a .80 en claridad, relevancia y representatividad. En estos dos últimos aspectos se obtuvieron los coeficientes más altos, en todos los casos superiores a .90. Estos resultados permiten concluir que, las dimensiones consideradas cuentan con evidencia de validez basada en el contenido de los ítems que las componen.

Tabla 6.

Coeficientes $V$ de Aiken promedio para las variables de la escala PROTO HM de interacción con los objetos.

\begin{tabular}{|c|c|c|c|}
\hline Variable & Claridad & Relevancia & Representatividad \\
\hline Dimensión I: Permanencia del objeto & .936 & .983 & 1.000 \\
\hline Dimensión II: Inteligencia práctica & .885 & .944 & .952 \\
\hline Estrategias medio-fin & .900 & .953 & .960 \\
\hline $\begin{array}{l}\text { Estrategias de anticipación } \quad \text { y } \\
\text { planificación }\end{array}$ & .867 & .933 & .942 \\
\hline $\begin{array}{l}\text { Dimensión III: Relaciones entre los } \\
\text { objetos }\end{array}$ & .910 & .960 & .960 \\
\hline
\end{tabular}

\section{Discusión}

Considerando la importancia de poder medir el desarrollo de las habilidades protomentalistas, precursoras de la teoría de la mente que es la base cognitiva de la empatía (Riviére, 2000; Sáiz, Carbonero \& Román, 2012) se construyó y se obtuvo evidencias de validez de contenido de la Escala para la Evaluación del Desarrollo de Habilidades Protomentalistas (PROTO - HM) esto a partir de identificar la escasez y los problemas para obtener instrumentos que evalúen este constructo en poblaciones de la primera infancia. A la fecha, no se encontraron estudios que informaran evidencias de la existencia de instrumentos para la evaluación de habilidades protomentalistas en el ámbito nacional, coincidiendo con Westby y Robinson (2014) quienes manifiestan que en la actualidad la existencia de herramientas estandarizadas disponibles para evaluar las dimensiones de las habilidades protomentalistas son escasas y aún menos estudiadas desde un enfoque más psicométrico.

La Escala del desarrollo de Habilidades Protomentalistas (PROTO - HM), en su versión original aporta evidencias de validez de contenido bastante significativas, tanto en las habilidades de interacción con las personas como en habilidades de interacción con los objetos, lo que nos permite afirmar que el contenido de los ítems se ajusta a las dimensiones a las que pertenecen y al constructo que miden. A su vez evidencia un buen funcionamiento de ítems debido a que los 
límites inferiores del intervalo de confianza al 95\%, fueron mayores a .50 en todos los casos (Penfield \& Giacobbi, 2004). Es oportuno precisar que la validación psicométrica de un instrumento involucra que este evidencie características básicas que son requeridas para que pueda utilizarse en una población específica de manera que se asegure que los resultados de sus mediciones son éticas, precisas, exactas y estables (Sánchez \& Echeverry, 2004).

Por otro lado, cabe mencionar que el criterio de claridad de las subdimensiones de Atención e Intersubjetividad primaria y del ítem 13 de la subdimensión Estrategias de anticipación y planificación fueron observados para su reestructuración, no obstante, estos valores fueron superiores a .70 que se encuentra en el límite permitido (Aiken, 1985). A partir de este juicio de expertos se realizaron algunas adaptaciones para mejorar el grado de claridad de algunos ítems que permitieron una mejor comprensión de la demanda de los reactivos por parte de los evaluadores y a su vez se procedió a la contextualización y adaptación de la escala a la realidad en la que se realizó el presente estudio y al grupo etario a la que va dirigida.

Esto refleja que la escala PROTO-HM es un instrumento que cuenta con evidencias de validez de contenido, pero que aún requiere examinar de forma más extensa las propiedades psicométricas del instrumento, lo cual es una limitación por el momento. Sin embargo, una vez culminado el proceso psicométrico, este instrumento es candidato para poder realizar perfiles poblacionales, establecer valores de referencia para estudios posteriores sobre desarrollo infantil, evaluar el efecto de intervenciones en habilidades protomentalistas para orientar acciones y decisiones de política pública en salud psicológica de la primera infancia y realizar estudios de investigación en otros usos que presenta la correcta valoración de este constructo.

\section{Conclusiones}

El análisis psicométrico de validez demuestra que la Escala para la Evaluación del Desarrollo de Habilidades Protomentalistas (PROTO - HM) es un instrumento que se ajusta al modelo conceptual en el que se basa la investigación (de Villiers \& de Villiers, 2014; Riviére, 2000; Sáiz, Carbonero y Román, 2012; Westby \& Robinson, 2014).

Los ítems de la escala PROTO HM de interacción con las personas, evidencian claridad, relevancia y representatividad debido a que todos ellos presentaron valores $\mathrm{V}$ de Aiken superiores a .80 , lo que nos permite afirmar que el contenido de los ítems se ajusta a las dimensiones a las que pertenecen y al constructo que miden a su vez evidencia un buen funcionamiento de ítems debido a que los límites inferiores del intervalo de confianza al $95 \%$, fueron mayores a .50 en todos los casos.

Las dimensiones y subdimensiones de la Escala PROTO - HM de interacción con las personas obtuvieron coeficientes $\mathrm{V}$ de Aiken por encima de .90 , con excepción de las subdimensiones Atención e Intersubjetividad primaria ambas respecto a la claridad de sus ítems, no obstante, estos valores fueron superiores a .80, considerándose un nivel adecuado en especial en relevancia y representatividad respecto al constructo que miden.

Los ítems de la escala PROTO HM de interacción con los objetos, evidencian un nivel adecuado en claridad, relevancia y representatividad debido a que todos ellos presentaron coeficientes $\mathrm{V}$ de Aiken por encima de .80, únicamente el ítem 13 de la subdimensión Estrategias de anticipación y planificación presentó un coeficiente $\mathrm{V}=.733$, estando dentro de los límites permitidos (superior a .70), lo que nos permite afirmar que el contenido de los ítems se ajusta a las dimensiones a las que pertenecen y al constructo que miden, a su vez evidencia un buen funcionamiento de ítems debido a que los límites inferiores del intervalo de confianza al 95\%, fueron mayores a .50 en todos los casos.

Las dimensiones y subdimensiones de la escala PROTO - HM de interacción con los objetos obtuvieron coeficientes $\mathrm{V}$ de Aiken superiores a .80 en claridad, relevancia y 
representatividad. En estos dos últimos aspectos se obtuvieron los coeficientes más altos, V de Aiken superiores a .90 en todos los casos.

\section{Referencias}

Adolph, K. E., Tamis-LeMonda, C. S., Ishak, S., Karasik, L. B. \& Lobo, S. A. (2008). Locomotor experience and use of social information is posture specific. Developmental Psychology, 44 (6), 1705-1714. doi: https://doi.org/10.1037/a0013852

Aiken, L. (1985). Three coefficients for analyzing the reliability and validity of ratings. Educational and Psychological Measurement, 45, 131-142. doi: https://doi.org/10.1177/0013164485451012

Astington, J. W., \& Dack, L. A. (2008). Theory of mind. In: Haith MM, Benson JB, eds. Encyclopedia of infant and early childhood development, 3, 343-356. San Diego, CA: Academic Press.

Astington, J. W., \& Edward, M. J. (2010). El desarrollo de la teoría de la mente en la primera infancia. En: Tremblay, RE., Boivin, M. \& Peters RDeV. Eds. PD, Zelazo tema ed. eds. Encyclopedia of infant and early childhood development. Recuperado de http://www.child-encyclopedia.com/sites/default/files/textes-experts/en/588/thedevelopment-of-theory-of-mind-in-early-childhood.pdf

Ato, M., López, J., \& Benavente, A. (2013). Un sistema de clasificación de los diseños de investigación en psicología. Anales de Psicología, 29(3), 1038-1059. doi: https://doi.org/10.6018/analesps.29.3.178511

Baldwin, D. A., \& Moses, L. J. (2001). Links between social understanding and early word learning: Challenges to current accounts. Social Development, 10(3), 309-329. doi: https://doi.org/10.1111/1467-9507.00168

Berk, L. E. (2004). Desarrollo del niño y del adolescente. Madrid: Pearson.

Brooks, R., \& Meltzoff, A. N. (2005). The development of gaze following and its relation to language. Developmental Science, 8(6), 535-543. doi: https://doi.org/10.1111/j.14677687.2005.00445.x

Chopik, W., O'Brien, E., \& Konrath, S. (2017). Differences in empathic concern and perspective taking Across 63 Countries. Journal of Cross-Cultural Psychology, 48(1), 23-38. doi: https://doi.org/10.1177/0022022116673910

Davis, M. H. (1994). Empathy: A social psychological approach. Boulder, CO: Westview Press. Recuperado de: https://content.taylorfrancis.com/books/download?dac=C2017-076734-2\&isbn=9780429962448\&format=googlePreviewPdf

Decety, J., \& Lamm, C. (2006). Human empathy through the lens of social neuroscience. The Scientific World Journal, 6, 1146-1163. doi: http://dx.doi.org/10.1100/tsw.2006.221

de Villiers, J.G. \& de Villiers, P.A. (2014). The role of language in theory of mind development. Topics in Language Disorders, 34 (4), 313-328. Recuperado de https://alliedhealth.ceconnection.com/files/TheRoleofLanguageinTheoryofMindDevel opment-1415277302473.pdf

Escobar-Pérez, J., \& Cuervo-Martínez, A. (2008). Validez de contenido y juicio de expertos: Una aproximación a su utilización. Avances en Medición, 6, 27-36. Recuperado de: http://www.humanas.unal.edu.co/psicometria/files/7113/8574/5708/Articulo3_Juicio_ de_expertos_27-36.pdf

Herold, K., \& Akhtar, N. (2008). Imitative learning from a third -party interaction: Relations with self-Journal of recognition and perspective taking. Experimental Child Psychology, 101, 114-123.

Instituto Nacional de Estadística e Informática (2018). Boletín de estadísticas de seguridad ciudadana. Informe Técnico 4. Lima - Perú: INEI. Recuperado de https://www.inei.gob.pe/media/MenuRecursivo/boletines/04-informe-tecnicon04_estadisticas-seguridad-ciudadana-ene-jun2018.pdf

Kimbi, Y. (2014). Theory of mind abilities and deficits in autism spectrum disorders. Topics in Language Disorders, 34(4), $329 \quad-\quad 343 . \quad$ doi: https://doi.org/10.1097/TLD.0000000000000033 
Lowly, L. (2016). Tuning in to Others: How Young Children Develop Theory of Mind. The Hanen Centre. Recuperado de: http://www.hanen.org/Helpful-Info/Articles/Tuning-In-toOthers-How-Young-Children-Develop.aspx

Merino, C., \& Livia, J. (2009). Intervalos de confianza asimétricos para el índice de validez de contenido: Un programa Visual Basic para la V de Aiken. Anales de Psicología, 25, 169-171.

Miller, S. (2010). Social-cognitive development in early childhood. In B. R. Tremblay, M. Boivin \& R. DeV. Peters (Eds.), Encyclopaedia in an early childhood development (pp. 1-5). Montreal: Montreal Centre of excellence for Early Childhood.

Miller, S. (2012). Theory of mind: Beyond the preschool years. New York, N. Y.: Psychology Press. doi: https://doi.org/10.4324/9780203122730

Moll, H., \& Tomasello, M. (2007). How 14 - and 18- Month- old know what others have experienced. Developmental Psychology, 43(2), 309-317. doi: https://doi.org/10.1037/0012-1649.43.2.309

Moore, C. (2010). Social Cognition in infancy. Encyclopedia on Early Childhood Development. Recuperado de http://www.child-encyclopedia.com/sites/default/files/textesexperts/en/588/social-cognition-in-infancy.pdf

Penfield, R., \& Giacobbi, P. Jr. (2004) Applying a score confidence interval to Aiken's item content-relevance index. Measurement in Physical Education and Exercise Science, 8, 213-225.

Peterson, C. C., Wellman, H. M., \& Slaughter, V. (2012). The mind behind the message: Advancing Theory of mind scales for typically developing children, and those with deafness, autism, or Asperger syndrome. Child Development, 83(2), 469-485. doi: https://doi.org/10.1111/j.1467-8624.2011.01728.x

Riviére, A., \& Martos, J. (1997). El tratamiento del Autismo. Nuevas perspectivas. Madrid: INSERSO.

Riviére, A. (2000). Intencionalidad y metarrepresentación: una perspectiva evolutiva. En J.M. Ruiz-Vargas \& M. Belinchón (Eds.), Ángel Riviére Obras escogidas. 3, Metarrepresentación y semiosis (1-6). Madrid: Panamericana.

Rochat, P. (2001). The infant's world. Cambridge, MA: Harvard University Press.

Sáiz, M. C., \& Román, J. M. (2011). Estimulación mentalista en la Primera Infancia. Madrid: CEPE.

Sáiz, M. C., Carbonero, M. A., \& Román, J. M. (2012). Investigación y formación de profesorado en el aula: desarrollo de habilidades proto-mentalistas en alumnos de escuela infantil con necesidades educativas especiales. REIFOP, 15(1), 27-36. Recuperado de https://dialnet.unirioja.es/servlet/articulo?codigo $=4616597$

Sánchez, R., \& Echeverry, J. (2004). Validación de Escalas de Medición en Salud. Revista de Salud Pública, 6(3), 302-318.

Skjong, R., \& Wentworth, B. H. (2001). Expert Judgement and risk perception. Proceedings of the International Offshore and Polar Engineering Conference, 4, 537-544. Retrieved from: https://www.researchgate.net/publication/286613666_Expert_judgment_and_ris k_perception

Sommerville, J. A. (2010). Infants' Social Cognitive Knowledge. In: Tremblay RE, Boivin M, Peters RDeV, eds. Zelazo PD, topic ed. Encyclopedia on Early Childhood Development [online]. Recuperado de http://www.childencyclopedia.com/sites/default/files/textes-experts/en/588/infants-social-cognitiveknowledge.pdf

Sussman, F. (2006). Talkability - People skills for verbal children on the autism spectrum: A guide for parents. Toronto, ON: Hanen Eartly Language Program.

Warneken, F., \& Tomasello, M. (2009). Varieties of altruism in children and chimpanzees. Trens in Cognitive Science. doi: https://doi.org/10.1016/j.tics.2009.06.008

Wellman, H. M., \& Liu, D. (2004). Scaling theory of mind tasks. Child Development, 75, 759763. 
Westby, C., \& Robinson, L. (2014). A developmental perspective for promoting theory of mind.
Topics
in Language
Disorders, 34(4),
362-383.
doi:

https://doi.org/10.1097/TLD.0000000000000035

\section{Financiación}

Recursos en especie por el Vicerrectorado de Investigación y Posgrado de la Universidad Nacional Mayor de San Marcos de Lima - Perú. 